\title{
Reliability of the Supraspinatus Muscle Thickness Measurement by Ultrasonography
}

\author{
Tae Im Yi, M.D., In Soo Han, M.D., Joo Sup Kim, M.D., Ju Ryeon Jin, M.D., Jea Shin Han, M.D.
}

Department of Rehabilitation Medicine, Bundang Jesaeng General Hospital, Seongnam 463-774, Korea

Objective To assess the intrarater and interrater reliability of the supraspinatus thickness measured by ultrasonography (US) in normal subjects and to identify the relationship between the supraspinatus thickness measured by US and cross sectional area (CSA) of the supraspinatus muscle by magnetic resonance imaging (MRI) in hemiplegic patients.

Method We examined 20 shoulders of normal subjects and 10 shoulders of hemiplegic patients. In normal subjects, one examiner measured the supraspinatus thickness twice by US at the scapular notch and another examiner measured the supraspinatus thickness several days later. The intrarater and interrater reliability of supraspinatus thickness measurements were then evaluated. In hemiplegic patients, the supraspinatus thickness at the scapular notch was measured by US in affected side and compared with CSA of the supraspinatus muscle at the scapular notch and the Y-view of MRI.

Results One examiner's supraspinatus thickness measurement average was $1.72 \pm 0.21 \mathrm{~cm}$ and $1.74 \pm 0.24 \mathrm{~cm}$, and the other examiner's supraspinatus thickness measurement average was $1.74 \pm 0.22 \mathrm{~cm}$ in normal subjects. Intraclass correlation coefficients of intrarater and interrater examination were 0.91 and 0.88 , respectively. For hemiplegic patients, the supraspinatus thickness measured by US was $1.66 \pm 0.13 \mathrm{~cm}$ and CSA by MRI was $4.83 \pm 0.88 \mathrm{~cm}^{2}$ at the Y-view and $5.61 \pm 1.19 \mathrm{~cm}^{2}$ at the scapular notch. The Pearson Correlation Coefficient between the supraspinatus thickness at the scapular notch and the CSA at the Y-view was 0.72 and that between the supraspinatus thickness and CSA at the scapular notch was 0.76 .

Conclusion The supraspinatus thickness measurement by US is a reliable method and is positively correlated with the CSA of the supraspinatus muscle in MRI in hemiplegic patients. Therefore, supraspinatus thickness measurement by US can be used in the evaluation of muscle atrophy and to determine therapeutic effects in hemiplegic patients.

Key Words Supraspinatus, Thickness, Reliability, Ultrasonography, Magnetic resonance imaging

Received May 2, 2012; Accepted June 13, 2012

Corresponding author: In Soo Han

Department of Rehabilitation Medicine, Daejin Medical Center Bundang Jesaeng General Hospital, 255-2, Seohyeon-dong, Bundang-gu, Seongnam 463-774, Korea

Tel: +82-31-779-0395, Fax: +82-31-779-0635, E-mail: hislyw@gmail.com

(c) This is an open-access article distributed under the terms of the Creative Commons Attribution Non-Commercial License (http:// creativecommons.org/licenses/by-nc/3.0) which permits unrestricted noncommercial use, distribution, and reproduction in any medium, provided the original work is properly cited.

Copyright $\odot 2012$ by Korean Academy of Rehabilitation Medicine

\section{INTRODUCTION}

The glenohumeral joint shows the greatest amount of motion of any joint in the human body. ${ }^{1}$ The rotator cuff provides stability by compressing the humeral head within the glenoid fossa. ${ }^{2}$ If pathologic lesions of the rotator cuff or hemiplegia are present, the affected glenohumeral joint is destabilized and complications develop. Lesions 
of the rotator cuff that include the supraspinatus muscle often develop because of an overuse or due to traumatic injury. ${ }^{3}$ Because lesions of the rotator cuff may be accompanied by muscle atrophy with fatty infiltration that is progressive and probably irreversible, precise assessment of the affected rotator cuff may be crucial in establishing a therapeutic plan and predicting patient prognosis. ${ }^{4,5}$

We can assess the degree of supraspinatus activity using electromyography. The relative contribution of the supraspinatus muscle to the rotator cuff during maximal voluntary isometric muscle strength measurement can be assessed after anesthetizing the suprascapular nerve at proximal and distal sites and degree of the supraspinatus muscle atrophy can be evaluated by using imaging methods such as magnetic resonance imaging (MRI).,6-14 However, methods such as electromyography and selective nerve blocks are invasive and painful. Therefore, imaging methods are preferable for evaluating the supraspinatus muscle.

Imaging methods used to assess the supraspinatus muscle include MRI, computed tomography (CT), and diagnostic ultrasonography (US). MRI is considered to be the best method for obtaining clear images of soft tissue. ${ }^{15}$ Yanagisawa et al. ${ }^{13}$ reported that the supraspinatus muscle at the scapular notch has the largest cross sectional area (CSA) and Lieber reported that muscle size has a pronounced influence on muscle function. ${ }^{16}$ Therefore, measuring the CSA of the supraspinatus muscle at the scapular notch may be useful in evaluating the muscle's atrophy and function.

However, MRI is expensive and not always available. In contrast, US is less expensive than MRI and is typically available in outpatient clinics. However, evaluation of the supraspinatus muscle at the scapular notch by US is complicated because a clear image of the muscle is difficult to obtain because of acoustic shadowing by the clavicle and scapular spine. Therefore, obtaining a CSA of the supraspinatus muscle by US can be influenced by subjective factors, and the degree of the supraspinatus atrophy can be interpreted differently by examiners. ${ }^{17}$ Consequently, the reliability of US examination is low and its clinical use is limited.

The aim of this study was to assess the intrarater and interrater reliability of the supraspinatus thickness measurements obtained by US and to indentify the association between the supraspinatus thickness measured by
US and the CSA of the supraspinatus muscle determined by MRI.

\section{MATERIALS AND METHODS}

\section{Subjects}

Twenty supraspinatus muscles in ten asymptomatic subjects were assessed in this study to identify the intrarater and interrater reliability in measuring the supraspinatus muscle by US. Subjects who had shoulder pain and a history of surgery or who had an ultrasonographic abnormality and a difficulty in using their upper extremities in activities of daily living were excluded from the study. In addition, ten stroke patients with hemiplegia who were admitted to an inpatient rehabilitation unit were included to assess the relationship of the supraspinatus thickness measured by US and the CSA measured by MRI. In these stroke patients, ten supraspinatus thicknesses and the CSA of the hemiplegic side were measured. The study was performed prospectively after explaining the purpose and content of the study to the patients.

\section{Methods}

In this study, we used the Korean version of Modified Bathel Index (K-MBI) to assess the patients' ADLs and we used the Motricity Index (MI) to measure the abduction of the affected shoulder. The K-MBI comprised 10 items, including personal hygiene, bathing, feeding, toileting, stairs up and down, dressing, defecation, voiding, ambulation, and bed transfer, for 100 points. The MI assesses shoulder abduction when moving the flexed elbow from the chest and is composed of $0,9,14,19,25,33$ points according to the abduction: a score of 0 indicates no movement, 19 indicates movement against gravity but not against resistance, and 33 indicates normal movement. ${ }^{18}$ We then measured the supraspinatus thickness by US and the CSA of the supraspinatus muscle by MRI.

\section{Measurement of the supraspinatus thickness by ultra-} sonography: All normal subjects sat in a chair with their shoulders in a neutral position and their elbows in full extension. We palpated the scapular spine and drew a line on the surface of body along the scapular spine. We also drew a line along the acromion with same method and denoted the acromion angle where two lines met. We palpated and marked the coracoid process and connected the acromion angle and coracoid process (AC 
line) (Fig. 1-A).

The US was performed by two physiatrists using a linear probe of 5-12 MHz LOGIQ E9 (General Electric Healthcare, Milwaukee, USA). We identified the scapular notch after locating the probe parallel to the action of the muscle. ${ }^{11}$ The probe was rotated parallel to the AC line, with
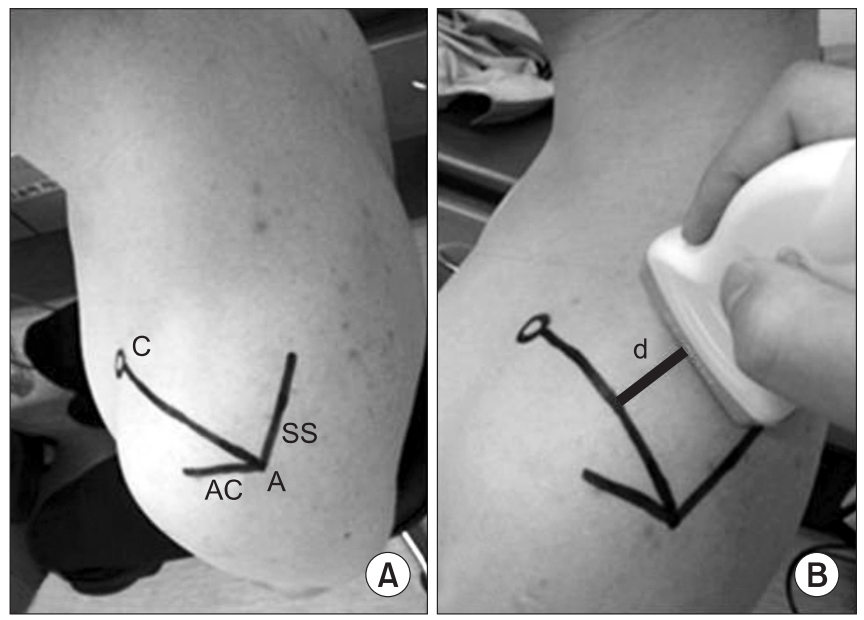

Fig. 1. Evaluation of the supraspinatus muscle using ultrasonography. Surface anatomy of left shoulder (A) and placement of ultrasonographic probe (B). We identified the scapular notch on the monitor in parallel to the action of supraspinatus muscle and rotated the probe parallel to the AC line. After we observed cross section of the supraspinatus muscle on the monitor, We calculated the distance (d) between the AC line and the lateral side of the probe. C: Coracoid process, AC: Acromion, SS: Scapular spine, A: Acromial angle, AC line: Line between A and C, d: The closest distance between AC line and lateral side of ultrasonographic probe.
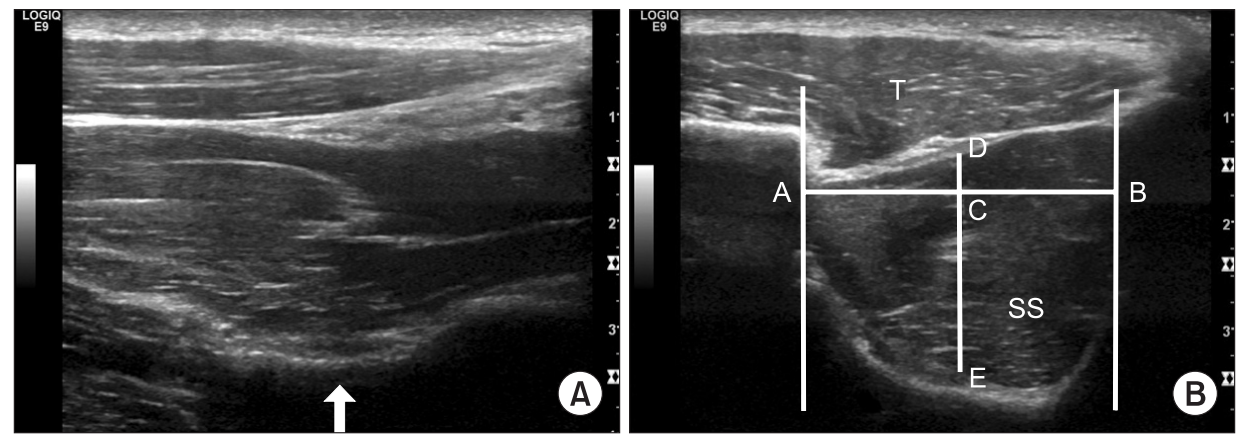

Fig. 2. Evaluation of the supraspinatus muscle using ultrasonography. Ultrasonographic findings of supraspinatus muscle which is parallel to the muscle line of action (A) and parallel to AC line (B) in normal subject. The arrow indicates scapular notch. AC line: Line between acromial angle and coracoids process, SS: Supraspinatus, T: Trapezius, A: Acoustic shadowing of clavicle, B: Acoustic shadowing of scapular spine, C: Mid-point of distance between A and B, D: Upper border of supraspinatus muscle, E: Lower border of supraspinatus muscle. Distance between D and E corresponds with supraspinatus muscle thickness in this study.

scapular notch placed at the center of monitor. When we identified the cross section of the supraspinatus muscle, we calculated the closest distance between the AC line and the lateral side of the probe (Fig. 1-B).

We used the acoustic shadow of the clavicle as an anterior border and that of the scapular spine as a posterior border. The closest borders of the supraspinatus muscle from the center of muscle were used as the superior and inferior borders to measure the supraspinatus thickness. ${ }^{11}$ We measured the closest horizontal distance between the acoustic shadowing of the clavicle and the scapular spine and obtained the median of that distance. We then measured the closest vertical distance between the high echogenic line of the superior and inferior borders of the supraspinatus muscle from this median and from the center of muscle (Fig. 2-B). Marks made on the skin were removed after the examination.

A few days later, we measured the thickness of the same supraspinatus muscle to assess the intrarater reliability. We moved the probe parallel from the AC line to a degree such as ' $d$ ' to measure the initial evaluation, and we observed the cross section of the supraspinatus muscle and measured the supraspinatus thickness at that cross section. The other examiner, who was unaware of the previous results, measured the supraspinatus thickness with the same method to evaluate the interrater reliability.

If the compressing pressure of the probe was too strong when we measured the supraspinatus thickness by US, this could compromise the supraspinatus thickness. Consequently, in this study, we kept the compressing pressure of the probe at a minimum so as not to influence the 
trapezius and supraspinatus muscle when we placed the probe at the $\mathrm{AC}$ line.

The supraspinatus thickness of the affected side in hemiplegic patients was measured using the same method described for the initial evaluation.

Measurement of cross sectional area of the supraspinatus muscle by MRI: We made measurements on ten hemiplegic patients using a 1.5T MR system Symphony (Siemens, Erlagen, Germany). The subjects were placed supine with their arms at the sides of their bodies and their elbows extended. We acquired consecutive T1wieghted images of the affected shoulder at $3.5 \mathrm{~mm}$ intervals from glenoid surface to the medial border of the scapula. The affected supraspinatus muscles were classified using the five stages proposed by Goutallier et al. ${ }^{19}$ to identify atrophy of the supraspinatus muscle. Each CSA of the supraspinatus muscle was measured at the position composed of a Y shaped structure where the coracoid process and scapula spine met (Y-view) and at scapular notch (Fig. 3).

We used the NUMARIS/4 (version: syngo MR A30) software and the CSA of the muscles was obtained using a tracing method.

We used ANOVA to compare the intrarater and interrater differences and the Intraclass Correlation Coefficient (ICC) was used to assess the intrarater and interrater correlation in normal subjects. The Pearson Correlation Coefficient (PCC) was obtained to identify the correlation of the supraspinatus thickness at the scapular notch measured by US and CSA at the Y-view and by MRI at the scapular notch.

Statistical analysis was conducted using Windows SPSS version 18.0 and the null hypothesis was rejected at $\mathrm{p}<0.05$.

\section{RESULTS}

Normal subjects included 4 men and 6 women with an average age of $23.80 \pm 1.47$ years, an average height of $166.90 \pm 7.72 \mathrm{~cm}$, and an average weight of $56.20 \pm 7.85 \mathrm{~kg}$. The hemiplegic patients included 9 men and 1 woman with an average age of $58.90 \pm 9.23$ years, an average height of $164.90 \pm 5.15 \mathrm{~cm}$, and an average weight of $62.50 \pm 4.64$ $\mathrm{kg}$. The average duration of the hemiplegia was $8.10 \pm 7.24$ months. Of the 10 hemiplegic patients, 5 had paralysis of the right side and 5 had paralysis of the left side. The

Table 1. General Characteristics of Subjects

\begin{tabular}{lcc}
\hline \multicolumn{1}{|c}{ Variables } & $\begin{array}{c}\text { Normal } \\
\text { subjects }\end{array}$ & $\begin{array}{c}\text { Hemiplegic } \\
\text { patients }\end{array}$ \\
\hline Age (years) & $23.80 \pm 1.47$ & $58.90 \pm 9.23$ \\
\hline Sex & & \\
$\quad$ Male & 4 & 9 \\
\hline Female & 6 & 1 \\
\hline Height (cm) & $166.90 \pm 7.72$ & $164.90 \pm 5.15$ \\
\hline Weight (kg) & $56.20 \pm 7.85$ & $62.50 \pm 4.64$ \\
\hline $\begin{array}{l}\text { Post stroke duration } \\
\text { (months) }\end{array}$ & $8.10 \pm 7.24$ \\
\hline Affected side & & \\
\hline Right & & \\
\hline Left & & 5 \\
\hline K-MBI & & $66.50 \pm 14.03$ \\
\hline MI & & $17.70 \pm 5.58$ \\
\hline $\begin{array}{l}\text { Goutallier's classification } \\
\text { of shoulder }\end{array}$ & \\
\hline Grade 1 & & \\
\hline Grade 2 & & 6 \\
\hline
\end{tabular}

Values are means \pm standard deviation

K-MBI: Korean version of Modified Barthel Index, MI: Motricity index
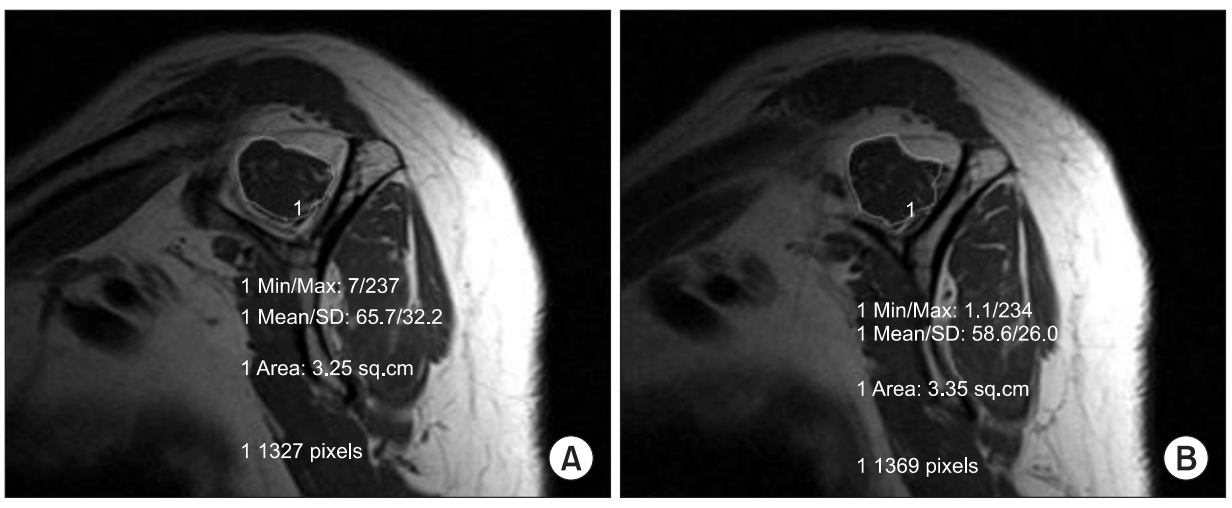

Fig. 3. Cross sectional area measurement of affected supraspinatus muscle by magnetic resonance imaging at the Y-view (A) and the suprascapular notch (B) in hemiplegic patients. 
average K-MBI was $66.50 \pm 14.03$ and the average MI was 17.70 \pm 5.58 . In total, 6 patients were Grade 1 and 4 patients were Grade 2, based on Goutallier's classification following MRI examination performed on the affected supraspinatus muscle (Table 1).

In normal subjects, the average supraspinatus thickness measured by US was $1.72 \pm 0.21 \mathrm{~cm}$ at the initial evaluation and $1.73 \pm 0.24 \mathrm{~cm}$ several days later. The average supraspinatus thickness measured by US performed by another examiner who did not know the previous results was $1.74 \pm 0.22 \mathrm{~cm}$. No significant differences were noted among the three examinations based on ANOVA. We also calculated ICC to assess the agreement among the three examinations. The intrarater ICC of the US examination performed by the same examiner was 0.91 and interrater ICC by another examiner was 0.88 (Table 2,3 ).

The average supraspinatus thickness measured by US in hemiplegic patients was $1.66 \pm 0.13 \mathrm{~cm}$. The average CSA of the supraspinatus muscle measured by MRI was $4.83 \pm 0.88 \mathrm{~cm}^{2}$ at the Y-view and $5.61 \pm 1.19 \mathrm{~cm}^{2}$ at the scapular notch. The PCC between the supraspinatus thickness measured by US at the scapular notch and the CSA of the supraspinatus muscle determined by MRI at the Y-view was 0.72 . The PCC between the supraspinatus thickness determined by US and CSA determined by MRI at the scapular notch was 0.76 (Table 2, 4).

\section{DISCUSSION}

Rotator cuff tears may be accompanied by muscle at-

Table 2. Thickness and Cross Sectional Area of the Supraspinatus Muscle in Normal Subjects and Hemiplegic Patients

\begin{tabular}{|c|c|c|}
\hline & $\begin{array}{l}\text { Normal } \\
\text { subjects }\end{array}$ & $\begin{array}{c}\text { Hemiplegic } \\
\text { patients }\end{array}$ \\
\hline \multicolumn{3}{|l|}{ Thickness by US (cm) } \\
\hline Test 1 & $1.72 \pm 0.21$ & $1.66 \pm 0.13$ \\
\hline Test 2 & $1.73 \pm 0.24$ & \\
\hline Test 3 & $1.74 \pm 0.22$ & \\
\hline \multicolumn{3}{|l|}{ CSA by MRI $\left(\mathrm{cm}^{2}\right)$} \\
\hline At the scapular notch & & $5.61 \pm 1.19$ \\
\hline At the Y-view & & $4.83 \pm 0.88$ \\
\hline
\end{tabular}

Values are mean \pm standard deviation

Test 1 and Test 2 are examined by same examiner and Test 3 is by another examiner

US: Ultrasonography, CSA: Cross sectional area, MRI: Magnetic resonance imaging rophy and fatty infiltration and may be progressive and irreversible. ${ }^{4,5}$ Consequently, assessment of muscle tears using imaging methods is crucial for determining the direction of therapy. ${ }^{11}$ Stroke can result in paralysis of the muscles of the affected side and paralysis of the supraspinatus muscle in the upper limb can cause subluxation of the shoulder joint. ${ }^{20}$ The prevalence of rotator cuff muscle as well as shoulder subluxation is high in hemiplegic patients. ${ }^{21}$ This complication can induce shoulder pain and negatively influence both the functional recovery of the upper extremity as well as rehabilitation exercise. For this reason, evaluation of the rotator cuff muscle, including the supraspinatus muscle, is essential.

De Ste Croix et al. ${ }^{22}$ reported that the maximal CSA was closely related to muscle function. Yanagisawa et al. ${ }^{13}$ used shoulder MRI to investigate the maximal CSA of the supraspinatus muscle and reported that the greatest CSA was measured at the scapular notch. Therefore, we considered the scapula notch to be a good site for measurement of the CSA for assessment of the supraspinatus muscle. However, because the cross section of the supraspinatus muscle cannot be clearly observed due to acoustic shadowing of the clavicle and scapular spine, measurement of the CSA of the supraspinatus muscle by US is difficult and the reliability of the examination can be further decreased by the subjectivity of the examiners. ${ }^{17}$ Our intention in the present study was therefore to try to minimize subjective factors in measuring the supraspinatus thickness by using surface anatomy and

Table 3. Reliability of the Supraspinatus Muscle Thickness Measurement Using Ultrasonography in Normal Subjects

\begin{tabular}{lccc}
\hline & ICC & $\begin{array}{c}\text { 95\% confidence } \\
\text { interval }\end{array}$ & p-value \\
\hline Intrarater reliability & 0.91 & $0.80-0.97$ & $<0.01$ \\
Interrater reliability & 0.88 & $0.72-0.95$ & $<0.01$ \\
\hline
\end{tabular}

ICC: Intraclass correlation coefficient

Table 4. Correlation between Supraspinatus Muscle Thickness Measured by Ultrasonography at the Scapular Notch and Cross Sectional Area Measured by MRI

\begin{tabular}{lcc}
\hline CSA measured by MRI & PCC & p-value \\
\hline At the scapular notch & 0.76 & 0.01 \\
At the Y-view & 0.72 & 0.02 \\
\hline
\end{tabular}

CSA: Cross sectional area, PCC: Pearson correlation coefficient, MRI: Magnetic resonance imaging 
acoustic shadowing.

The CSA of the supraspinatus muscle was measured by MRI by acquiring consecutive images from the glenoid surface to the medial border of the scapula, parallel to the glenoid surface. That is, measurement of cross section of the supraspinatus muscle was performed perpendicular to the direction of the scapula. Because the scapula was set out anteriorly 30 to 45 degrees from the coronal plane of the medial border of the scapula, the long axis of the probe was rotated 30 to 45 degrees from the sagittal plane, which allowed it to be placed perpendicular to the muscle line of action to identify the cross section of the supraspinatus muscle. ${ }^{23}$ In the present study, we set an AC line as a surface anatomical criterion that was perpendicular between the muscle line of action and the long axis of the probe. Because the average angle between the AC line and the sagittal plane was $33.50 \pm 6.68$ degrees in this study, our view was that if the long axis of the probe was placed parallel to the AC line, we could obtain the most perpendicular image to the muscle line of action.

When the supraspinatus muscle was assessed by US at the scapular notch, acoustic shadowing of the clavicle was made anteriorly and that of the scapular spine was made posteriorly. We measured the shortest perpendicular distance between the superior and inferior borders of the supraspinatus muscle based on the median between the two acoustic shadowings. We consider that subjective factors can be minimized in the examination because, when the US was performed, the surface anatomical site was constant and we measured the supraspinatus thickness using the acoustic shadowing of the clavicle and scapular spine at that position. The average intrarater ICC of the supraspinatus thickness, measured by surface anatomy and acoustic shadowing, was 0.91 and the interrater ICC was 0.88 . Therefore, our view is that when the supraspinatus thickness is measured by US, the use of surface anatomy and acoustic shadowing can improve the reliability of the examination.

The supraspinatus muscle has a three-dimensional structure, which means that measurement of the supraspinatus volume is needed for precise assessment of supraspinatus atrophy. ${ }^{17}$ This means that, ideally, the entire shoulder should be reconstructed by MRI to measure the supraspinatus volume, but this method is expensive and time-consuming. Lehtinen et al. ${ }^{15}$ reported a significant correlation between the supraspinatus volume and CSA and suggested that measurement of CSA is useful clinically. Juul-Kristensen et al. ${ }^{14}$ reported a significant correlation between the supraspinatus thickness and CSA measured by US and by MRI. However, in their study, the supraspinatus thickness was not measured at the maximal CSA but instead was determined at the middle of the supraspinatus muscle length, after the muscle length was measured at the distance between the medial border of the scapula and the lateral side of the acromion. A further limitation was that the US measurements were performed until about 1 year after the MRI. In addition, the supraspinatus muscle was not measured perpendicular to the muscle line of action but parallel to it.

Our aim in the current study was to measure the supraspinatus thickness by US at the site where the maximal CSA of the supraspinatus muscle was observed. Yanagisawa et al. ${ }^{13}$ reported that the CSA of the supraspinatus muscle is maximal at the scapular notch and Thomazeau et al. reported that the maximal CSA is found at the Yview. ${ }^{10}$ However, because of the difficulty in measuring the supraspinatus thickness by US at the Y-view, we measured the supraspinatus thickness by US at the scapular notch and assessed its correlation with the CSA measured at the scapular notch and Y-view by MRI. A strong positive correlation was evident by the PCC $(0.76)$ between the supraspinatus thickness measured by US and the CSA of the supraspinatus muscle measured by MRI at the scapular notch. The PCC between the supraspinatus thickness measured by US at the scapular notch and the CSA of the supraspinatus muscle measured by MRI at the Y-view was 0.72 . This means that the CSA of the supraspinatus muscle determined by MRI could be replaced with the supraspinatus thickness measured by ultrasonography. Therefore, measurement of the supraspinatus thickness by US at the scapular notch can be viewed as a useful tool for evaluating supraspinatus atrophy and function and may be helpful in monitoring therapeutic effects.

However, in this study, the sample size for the hemiplegic patients was small, at only 10 subjects, and atrophy of the hemiplegic supraspinatus was not highly variable (6 patients were Grade 1 and 4 were Grade 2, based on Goutallier's classification). Our view is that if the degree of atrophy of the supraspinatus muscle was more variable, more information about the relationship between 
the supraspinatus thickness and function would allow better validation of this tool.

\section{CONCLUSION}

Measurement of the supraspinatus thickness by US is a highly reliable intrarater and interrater method in normal subjects and is highly correlated with the CSA of the supraspinatus muscle in hemiplegic patients.

Therefore, when measuring the supraspinatus thickness, if the surface anatomy and acoustic shadowing proposed in this study are used, this will be helpful in evaluating the strength of the affected upper extremity as well as in predicting functional recovery in hemiplegic patients.

\section{REFERENCES}

1. Rockwood CA, Matsen FA. The shoulder. Philadelphia: Saunders, 1998, 233-276

2. Wilk KE, Arrigo C. Current concepts in the rehabilitation of the athletic shoulder. J Orthop Phys Ther 1993; 18: $365-378$

3. Reinold MM, Macrina LC, Wilk KE, Fleisig GS, Dun S, Barrentine SW, Ellerbusch MT, Andrews JR. Electromyographic analysis of the supraspinatus and deltoid muscles during 3 common rehabilitation exercises. J Athl Train 2007; 42: 464-469

4. Bjorkenheim JM. Structure and function of the rabbit's supraspinatus muscle after resection of its tendon. Acta Orthop Scand 1989; 60: 461-463

5. Thomazeau H, Boukobza E, Morcet N, Chaperon J, Langlais F. Prediction of rotator cuff repair results by magnetic resonance imaging. Clin Orthop Relat Res 1997; 344: 275-283

6. Chaco J, Wolf E. Subluxation of the glenohumeral joint in hemiplegia. Am J Phys Med 1971; 50: 139-143

7. Boettcher CE, Ginn KA, Cathers I. Which is the optimal exercise to strengthen supraspinatus? Med Sci Sports Exerc 2009; 41: 1979-1983

8. Takeda Y, Kashiwaguchi S, Endo K, Matsuura T, Sasa T. The most effective exercise for strengthening the supraspinatus muscle: evaluation by magnetic resonance imaging. Am J Sports Med 2002; 30: 374-381

9. Gerber C, Blumenthal S, Curt A, Werner CM. Effect of selective experimental suprascapular nerve block on abduction and external rotation strength of the shoulder. Shoulder Elbow Surg 2007; 16: 815-820

10. Thomazeau H, Rolland Y, Lucas C, Duval JM, Langlais F. Atrophy of the supraspinatus belly. Assessment by MRI in 55 patients with rotator cuff pathology. Acta Orthop Scand 1996; 67: 264-268

11. Khoury V, Cardinal E, Brassard P. Atrophy and fatty infiltration of the supraspinatus muscle: sonography versus MRI. AJR Am J Roentgenol 2008; 190: 11051111

12. Katayose M, Magee DJ. The cross-sectional area of suprapinatus as measured by diagnostic ultrasound. J Bone Joint Surg Br 2001; 83: 565-568

13. Yanagisawa O, Dohi M, Okuwaki T, Tawara N, Niitsu M, Takahashi H. Appropriate slice location to assess maximal cross-sectional area of individual rotator cuff muscles in normal adults and athletes. Magn Reson Med Sci 2009; 8: 65-71

14. Juul-Kristensen B, Bojsen-Møller F, Holst E, Ekdahl C. Comparision of muscle sizes and moment arms of two rotator cuff muscles measured by ultrasonography and magnetic resonance imaging. Eur J Ultrasound 2000; 11: 161-173

15. Lehtinen JT, Tingart MJ, Apreleva M, Zurakowski D, Palmer W, Warner JJ. Practical assessment of rotator cuff muscle volumes using shoulder MRI. Acta Orthop Scand 2003; 74: 722-729

16. Lieber RL. Skeletal muscle structure and function, 1st ed, Baltimore: Williams \& Wilkins, 1992, 1-48

17. Kim YS, Heo NY, Kim MW. The test-retest reliability of supraspinatus cross-sectional area measurement by sonography. Ann Rehabil Med 2011; 35: 524-528

18. Collin C, Wade D. Assessing motor impairment after stroke: a pilot reliability study. J Neurol Neurosurg Psychiatry 1990; 53: 576-579

19. Goutallier D, Postel JM, Bernageau J, Lavau L, Voisin MC. Fatty muscle degeneration in cuff ruptures. Preand postoperative evaluation by CT scan. Clin Orthop Relat Res 1994; 304: 78-83

20. Basmajian JV. Muscles alive: their functions revealed by electromyography, 4th ed, Baltimore: Williams \& Wilkins, 1978, 421-485

21. Yoon TS, Kim DH, Park JW, Kwon BS, Ryu KH, Lee HJ, Park NK, Shim JH. Causes of the hemiplegic shoulder pain. J Korean Acad Rehab Med 2010; 34: 158-162

22. De Ste Croix M, Deighan M, Armstrong N. Assessment 
and interpretation of isokinetic muscle strength during growth and maturation. Sports Med 2003; 33: 727743
23. Culham E, Peat M. Functional anatomy of the shoulder complex. Orthop Sports Phys Ther 1993; 18: 342350 